\title{
Orange-Red Emitting Phosphors Sm3+- Activated Borosilicate for near UV-based White LEDs
}

\author{
Hong $\mathrm{Yu}$ \\ Research Institute for New Materials Technology \\ Chongqing University of Arts and Science Chongqing, \\ China \\ fishred520@163.com
}

\author{
Jinlei Chen* \\ College of Materials and Chemical Engineering \\ Chongqing University of Arts and Science \\ Chongqing, China \\ chenjinlei513@163.com
}

\begin{abstract}
A new phosphor Sr3B2SiO8: Sm3+ has been synthesized via solid-state reaction process. Emission/excitation spectra and photoluminescence decay behaviors were investigated in detail. The phosphors Sr3B2SiO8: Sm3+ can be excited under the excitation of 405 nm, Sr3B2SiO8: Sm3+ could show orange-red emitting light which cover the region from 450 to $700 \mathrm{~nm}$. The intensity of Sr3B2SiO8: $\mathrm{Sm3}+$ phosphor increases with $\mathrm{Sm3}+$ ions adding, then gradually decreases as the doping concentration becomes higher than 0.03 , it occurs concentration quenching the possibility of energy transfer between $\mathrm{Sm3}+$ ions increases, we can roughly estimate the critical distance of energy transfer (Rc) according to the report of Blasse, after calculating, the Rc value is about 12.6 $\AA$. When the $\mathrm{Sm3}+$ at 0.03 mol, the chromaticity coordinates of $\mathrm{Sr} 3 \mathrm{~B} 2 \mathrm{SiO}$ : $\mathrm{Sm3}+$ is $(0.62,0.34)$ which closed to the standard CIE of red light $(0.67,0.33)$. The results showed that Sr3B2SiO8: Sm3+ phosphors may be promising phosphor candidate in UV white LEDs.
\end{abstract}

Keywords-Sr3B2SiO8: Sm3+; Luminescence; Phosphor; Energy Transfer; Lifetime

\section{INTRODUCTION}

White-light-emitting diodes (W-LEDs) have been paid more and more attention as the advantage of longer lifetime, higher rendering index, higher luminosity efficiency and lower energy consumption $[1,2]$. The most common method is realized by combining the blue light of GaN chips and the yellow emission of YAG: Ce3+ [3-5], however, the type of white color varies with the input power and a poor color rendering index $(\mathrm{Ra}<80)$. Many efforts have been made to overcome these mention above disadvantages, novel phosphor materials can be effectively excited by ultraviolet or blue light which can emit strong blue, green, red light, gained importance [6-8]. Tricolor phosphors with high stability and intense absorption in UV spectral region are in demand to meet the optimum requirements of w-LEDs.

Red emitting phosphor is one of the tricolor luminescent materials for white LEDs. Up to now, many researchers have been done on red or reddish-orange phosphors doped by $\mathrm{Eu} 3+, \mathrm{Sm} 3+, \mathrm{Bi} 2+$ and $\mathrm{Mn} 4+$ ions, commonly doped are Eu3+ and $\mathrm{Sm} 3+$ ions, they all have sharp excitation peaks at around $400 \mathrm{~nm}$ and narrow emissions at 580 630 nm, due to characteristic intraconfigurational $4 \mathrm{f}-4 \mathrm{f}$ transitions. As well known, the sharp $4 \mathrm{f}-4 \mathrm{f}$ transitions and the full width at half maximum are outmost filled 5s 2 and 5p6 orbitals. Such an inert host dependence of $\mathrm{Eu} 3+$ and $\mathrm{Sm} 3+$ leads to the excellent reproduction quality of optical properties of red phosphor especially in the process of mass production. Therefore, it is strongly expected that $\mathrm{Eu} 3+$ or $\mathrm{Sm} 3+$ would be ideal activator for red phosphor for white LEDs.

Usually, the Sm3+ ion has sharp excitation peak at $\sim 400 \mathrm{~nm}$ and shows narrow emissions due to characteristic intraconfigurational $4 \mathrm{f}-4 \mathrm{f}$ transitions of $\mathrm{Sm} 3+$ in the host. The luminescence signal of $\mathrm{Sm} 3+$ ion doped materials shows characteristic lines determined by the peculiar electronic structure of $\mathrm{Sm} 3+$ ion energy levels. Sm3+ doped materials have been intensively investigated due to its increasing application in various fluorescent devices, and the search for novel reddish orange emitting phosphors has stimulated a growing interest in Sm3+ doped materials.

Borosilicate was selected as host due to its advantages, such as stable physical and chemical properties, excellent thermal stability and easy to acquire. Commonly, it used to be the glass substrate material, in borosilicate system, attribute to adding of the boric acid, therefore, it can reduce the synthesis temperature of preparation of silicate, in addition, in ultraviolet region, borosilicate also has better absorption, borosilicate can applied as fluorescent material for LED according to this characteristic[9]. Recently, less research reported about this aspect, the respect of this region is worth investigating. In the composition of borosilicate, there including two elements (B, Si), generally speaking, B coordinated in the form of BO3 (triangle) and BO4 (tetrahedron) and Si coordinated in the form of silicon oxygen tetrahedron, different patterns of the B-Si connection could cause borosilicate present diversified borosilicate structure, boron silicate has good chemical stability which dues to the existence of the structure of $\mathrm{BO} 4$.

Therefore, boron silicate as substrate of luminescent material is a good choice as the result of its excellent chemistry and thermal stabilization, facile synthesis and cheap raw materials (H3BO3), so it has been extensively applied to phosphor for LED, since it can be efficiency exccited by LED chips, there have been a few reports recently about this materials applied in phosphors for white LED, as is known to all, $\mathrm{Sr} 3 \mathrm{~B} 2 \mathrm{SiO} 8$ as a stable 
boron silicate host, the luminescence property with $\mathrm{Sm} 3+$ have not yet been investigated in detail. Therefore, in the present work, in this paper, we utilize the advantages of borosilicate and choose $\mathrm{Sr} 3 \mathrm{~B} 2 \mathrm{SiO} 8$ as substrate of luminescent material, and doped with trivalent rare earth $\mathrm{Sm} 3+$ to analyze the luminescence properties under ultraviolet excitation conditions.

\section{EXPERIMENTAL}

\section{A. Preparation of Sr3-xB2SiO8: $x \mathrm{Sm} 3+(x=0.01 \sim 0.09)$ samples}

Sr3B2SiO8: Sm3+phosphors were prepared by a solidstate reaction technique. Analytical reagent grade (99.90\%) SrCO3、H3BO3、 $\mathrm{SiO} 2$, spectrographically pure $(99.99 \%)$ $\mathrm{Sm} 2 \mathrm{O} 3$ were employed as reactants. Reactant samples were first quantified by the stoichiometric ratio and then thoroughly mixed by grinding them in an agate mortar for 2 hours, and then transferred the samples into the corundum crucible and placed in a muff furnace at $600^{\circ} \mathrm{C}$ for $1 \mathrm{~h}$, then get out and grinding them in an agate mortar for 1 hour again, subsequently, firing at $1000^{\circ} \mathrm{C}$ for $3 \mathrm{~h}$. Finally, corundum crucibles were cooled to room temperature and the phosphor samples were obtained.

\section{B. Apparatus and measurements}

The powder X-ray diffraction (XRD) patterns were recorded with a Bruker D8-advance X-ray powder diffractometer with $\mathrm{CuK} \alpha$ radiation ( $\lambda=1.5406 \AA$ ), the operation voltage and current were maintained at $40 \mathrm{kV}$ and $40 \mathrm{~mA}$, respectively, a scan rate of $2 \%$ min was applied to record the patterns in the range of $2 \theta=10 \sim 60^{\circ}$. The excitation and emission spectra were measured by a Spectrofluorophotometer RF-5301PC series equipped with a $150 \mathrm{~W}$ Xenon lamp. The luminescence decay curves were obtained from a Lecroy Wave Runner 6100 Digital Oscilloscope $(1 \mathrm{GHz})$.All the experiments were performed at room temperature.

\section{RESULTS AND DISCUSSION}

\section{A. Characteristic analysis of XRD}

The phase purities and the crystal structures of the asprepared powder samples were characterized by XRD at room temperature. The XRD patterns of Sr3B2SiO8: $\mathrm{Sm} 3+$ phosphor samples with the $\mathrm{Sm} 3+$ concentrations are seen in Figure 1. All of the diffraction peaks are accord with Sr3B2SiO8 (JCPDS card No.32-1224). The samples are of single phase without any impurities. This indicates that doping of $\mathrm{Sm} 3+$ in $\mathrm{Sr} 3 \mathrm{~B} 2 \mathrm{SiO} 8$ host with such a small concentration has no other phase specific changes, the radii of $\mathrm{Sm} 3+$ ion is close to the radius of $\mathrm{Sr} 3+$, it preferably occupy the $\mathrm{Sr} 2+$ ion [10].

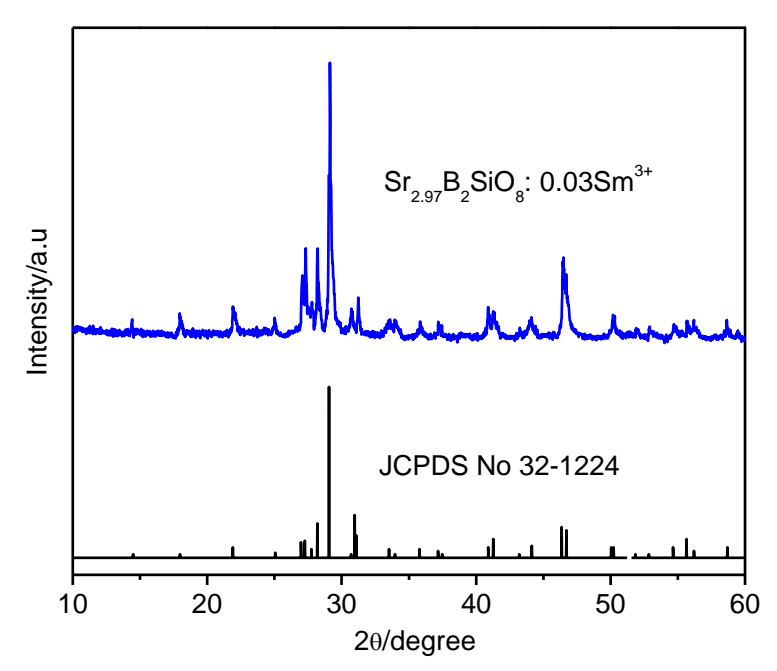

Figure 1. Typical XRD pattern of $\mathrm{Sr}_{2.97} \mathrm{~B}_{2} \mathrm{SiO}_{8}: 0.03 \mathrm{Sm}^{3+}$

\section{B. Photoluminescence of \\ Sr2.97B2SiO8:0.03Sm3 + phosphors}

PL excitation and emission spectra of $\mathrm{Sm} 3+$ doped Sr3B2SiO8 phosphor samples with 3 mol\% concentration are presented in Figure 2. Figure 2-a shows the excitation spectrum of the sample that monitored at the emission of $600 \mathrm{~nm}$ which consists the band in the range of 300 to 500 $\mathrm{nm}$ with the maximum at $405 \mathrm{~nm}$ which attributed the $6 \mathrm{H} 5 / 2 \rightarrow 4 \mathrm{~K} 11 / 2$ of Sm3+.Figure 2-b presents the emission spectrum of the sample excited by $405 \mathrm{~nm}$, which covers the region from 450 to $700 \mathrm{~nm}$ consisting of the characteristic transitions of Sm3+, namely, 4G5/2 $\rightarrow 6 \mathrm{H} 5 / 2$ $(565 \mathrm{~nm}), 4 \mathrm{G} 5 / 2 \rightarrow 6 \mathrm{H} 7 / 2(600 \mathrm{~nm})$ and $4 \mathrm{G} 5 / 2 \rightarrow 6 \mathrm{H} 9 / 2$ $(647 \mathrm{~nm})$, among these characteristic peaks of $\mathrm{Sm} 3+$, the emission of $4 \mathrm{G} 5 / 2 \rightarrow 6 \mathrm{H} 5 / 2(565 \mathrm{~nm})$ and $4 \mathrm{G} 5 / 2 \rightarrow 6 \mathrm{H} 7 / 2$ $(600 \mathrm{~nm})$ are predominant.

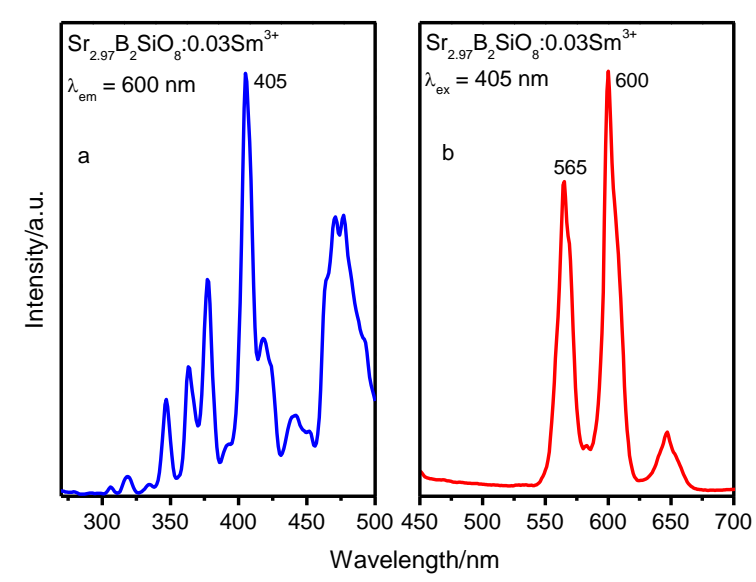

Figure 2. Photoluminescence excitation $(\mathrm{a}, \lambda \mathrm{em}=600 \mathrm{~nm})$ and emission (b, $\lambda$ ex $=405 \mathrm{~nm}$ ) spectra of $\mathrm{Sr}_{2.97} \mathrm{~B}_{2} \mathrm{SiO}_{8}: 0.03 \mathrm{Sm}^{3+}$ 
Figure 3 shows the emission intensity of Sr3B2SiO8: $\mathrm{Sm} 3+(\mathrm{x}=0.01 \sim 0.09)$ with different concentration under the excitation of $405 \mathrm{~nm}$. The emission intensities of all of the emission are enhanced significantly with the Sm3+ concentration increasing, then gradually decreases as the doping concentration becomes higher than 0.03, it occurs concentration quenching, therefore, the optimum doping concentration of $\mathrm{Sm} 3+$ is fixed at $3 \mathrm{~mol} \%$. The concentration quenching may be induced by cross relaxation processes in close $\mathrm{Sm} 3+-\mathrm{Sm} 3+$. When the $\mathrm{Sm} 3+$ concentration increases, the possibility of energy transfer between Sm3+ ions will also increase. According to the report of Blasse, we can roughly estimate the critical distance of energy transfer (Rc), and calculated as follows[11]:

$$
R c \approx 2\left(\frac{3 V}{4 \pi X c N}\right)^{1 / 3}
$$

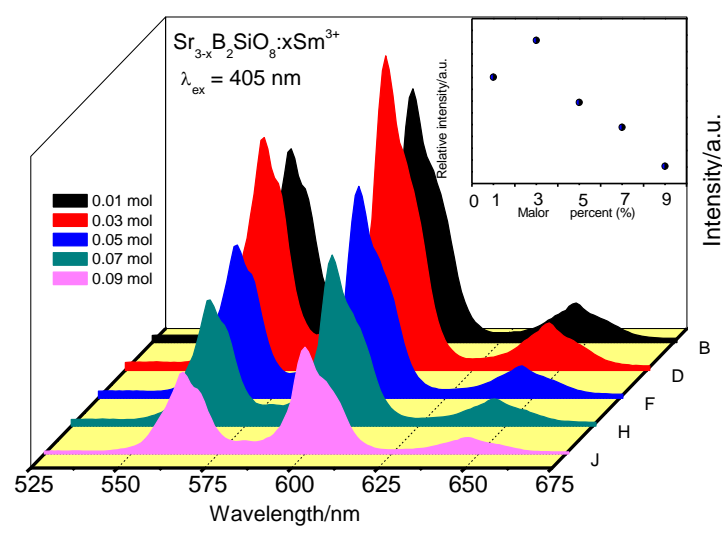

Figure 3. Photoluminescence emission spectra of $\mathrm{Sr} 3-\mathrm{xB} 2 \mathrm{SiO} 8: \mathrm{xSm} 3+$ with different $\mathrm{Sm} 3+$ concentration under the excitation of $405 \mathrm{~nm}$

Here $\mathrm{V}$ is the unit cell volume, $\mathrm{Xc}$ is the critical concentration, and $\mathrm{N}$ is the number of the ions in a unit cell. In Sr3B2SiO8, V=125 $\AA, \quad \mathrm{N}=4$, and the critical concentration $\mathrm{Xc}$ is about 0.03 in our system. The Rc value is about $12.6 \AA$ by using eq 1 .

In general, there are three mechanisms for nonradiate energy transfer, including exchange interaction, radiation reabsorption and electric multipolar interactions. The Rc obtained above indicate the little possibility of exchange interaction since the exchange interaction is predominant only for about 5-8 $\AA$ [12]. The mechanism of radiation is only efficacious when the fluorescence and absorption spectra are widely overlapping, which also does not applied in these cases. As a result, we can infer that the electric multipolar interactions would be responsible for the energy transfer mechanisms between the $\mathrm{Sm} 3+$ ions. As described by Dexter's energy transfer expressions of multipolar interaction and Reisfeld's approximation, the following relationship can be attained[13]:

$$
\frac{\eta s_{0}}{\eta s} \infty C^{\alpha / 3}
$$

where $\eta s_{0}$ and $\eta s$ represent the luminescence quantum efficiencies of the host with the absence and presence of $\mathrm{Sm} 3+$ ions. The value for $\alpha=6,8$ and 10 corresponds to dipole-dipole (d-d), dipole-quadrupole (d-q) and quadrupole-quadrupole (q-q) interactions, respectively. While, the value of $\eta s_{0} \eta s_{\text {is }}$ hard to obtain, it can be approximately calculated used by the value of $I s_{0} I s$, where $I s_{0}$ and $I S$ on behalf of the luminescence intensity of the host without and with the Sm3+ ions, respectively, therefore, the following relationship can be attained:

$$
\frac{I s_{0}}{I s} \infty C^{\alpha / 3}
$$

The relationship between $I s_{0} / I S$ and $C^{\alpha / 3}$ based on the above equation can be illustrated, the best linear relationship could be obtained when $\alpha=6$, indicating that the mechanism of energy transfer between $\mathrm{Sm} 3+$ ions is d$\mathrm{d}$ interaction.

\section{Photoluminescence lifetimes of Sr3B2SiO8:Sm3+ phosphors}

Experiment tests the fluorescence lifetime of different concentrations of $\mathrm{Sm} 3+$ in $\mathrm{Sr} 3 \mathrm{~B} 2 \mathrm{SiO} 8$ system. The Figure 4 shows the fluorescence decay curves and simple orbit transition of Sm3+, after fitting, the values A1, t1, A2, t2 listed in TABLE1, their decay curves can be well fitted by a double exponential function:

(4)

$$
I=A 1 \exp (-t / t 1)+A 2 \exp (-t / t 2)
$$

where $\mathrm{t} 1$ and $\mathrm{t} 2$ are two components of the luminescence lifetime, A1 and A2 are the fitting parameters. The lifetime could be evaluated by $\mathrm{t}=$ $(\mathrm{A} 1 \mathrm{t} 12+\mathrm{A} 2 \mathrm{t} 22) /(\mathrm{A} 1 \mathrm{t} 1+\mathrm{A} 2 \mathrm{t} 2)$, where $\mathrm{t}$ is defined as the average lifetime, the luminescence lifetime after fitting show in TABLE1.The luminescence lifetime of 0.01 0.09mol of Sm3+ are 6.985 E-7, 8.972 E-7, 6.116 E7, 6.111 E-7 and 5.370 E-7 s.

A schematic diagram for $\mathrm{Sm} 3+$-activated $\mathrm{Sr} 3 \mathrm{~B} 2 \mathrm{SiO} 8$ is displayed in the inset of Figure 4, under the excitation of $405 \mathrm{~nm}, \mathrm{Sm} 3+$ ions are excited from the $6 \mathrm{H} 5 / 2$ ground state to the $4 F 7 / 2$ excited state and then to the $4 G 5 / 2$ energy level by non-radiative relaxation, then, transfer occurs from $4 \mathrm{G} 5 / 2$ to the level of $6 \mathrm{H} 5 / 2,6 \mathrm{H} 7 / 2$ and $6 \mathrm{H} 9 / 2$, emitting 565, 600 and $647 \mathrm{~nm}$, respectively, the $600 \mathrm{~nm}$ is obviously stronger, which could also leading to the orangered visible light. 


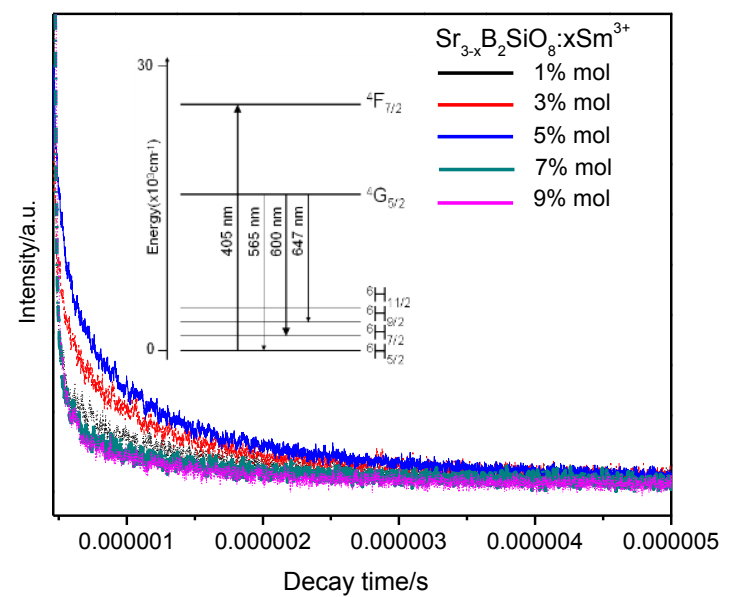

Figure 4. Photoluminescence decay curves of $\mathrm{Sm}^{3+}$ in $\mathrm{Sr}_{3 \mathrm{x}} \mathrm{B}_{2} \mathrm{SiO}_{8}$ : $\mathrm{xSm}^{3+}(\mathrm{x}=0.01,0.03,0.05,0.07,0.09)$ phosphors

TABLE I. THE FITTING PARAMETERS OF FLUORESCENCE LIFETIME OF SR3-XB2SIO8: X SM3+

\begin{tabular}{|l|c|c|c|c|c|}
\hline $\begin{array}{l}\mathrm{Sm}^{3+}(\mathrm{x} / \\
\mathrm{mol})\end{array}$ & $\mathrm{A}_{1}$ & $\mathrm{t}_{1}$ & $\mathrm{~A}_{2}$ & $\mathrm{t}_{2}$ & $\mathrm{t}$ \\
\hline 0.01 & 0.0002 & $1.220 \mathrm{E}-6$ & 0.0016 & $1.379 \mathrm{E}-7$ & $6.985 \mathrm{E}-7$ \\
\hline 0.03 & 0.0005 & $1.239 \mathrm{E}-6$ & 0.1238 & $9.378 \mathrm{E}-7$ & $8.972 \mathrm{E}-7$ \\
\hline 0.05 & 0.0005 & $1.247 \mathrm{E}-6$ & 0.1240 & $9.482 \mathrm{E}-7$ & $6.116 \mathrm{E}-7$ \\
\hline 0.07 & 0.0011 & $1.025 \mathrm{E}-6$ & 0.0473 & $1.172 \mathrm{E}-7$ & $6.111 \mathrm{E}-7$ \\
\hline 0.09 & 0.0012 & $1.209 \mathrm{E}-6$ & 0.0301 & $1.479 \mathrm{E}-7$ & $5.370 \mathrm{E}-7$ \\
\hline
\end{tabular}

\section{D. $\quad$ Chromaticity coordinates of Sr3B2SiO8: $\mathrm{Sm} 3+$ phosphors}

The chromaticity coordinates of different molar (0.01$0.09 \mathrm{~mol}$ ) of $\mathrm{Sm} 3+$-activated $\mathrm{Sr} 3 \mathrm{~B} 2 \mathrm{SiO} 8$ are presented in Figure 5. From Figure 5, we can observed that with the $\mathrm{Sm} 3+$ ions added (0.01-0.09 mol), the color moved to the red region, the chromaticity coordinates of $\mathrm{Sr} 3 \mathrm{~B} 2 \mathrm{SiO} 8$ : Sm3+are1:(0.55, 0.28),2: $(0.62,0.34), \quad 3:(0.61,0.32)$, $4:(0.59,0.30)$ and $5:(0.57,0.29)$, respectively. When the intensity of Sm3+ reach the maximum, it is close to the

\section{REFERENCES}

[1] H.K.Park, J.H.Oh, H.Kang, J.Zhang,Y.R.Do, "Hybrid 2D Photonic Crystal-Assisted Lu3A15O12:Ce Ceramic-Plate Phosphor and Free-Standing Red Film Phosphor for White LEDs with High Color-Rendering Index", Appl. Mater. Interfaces,vol.7,Feb.2015, doi: 10.1021/am507237n

[2] P.Lee,T.S.Chan,T.M.Chen,"NovelReddish-Orange-Emitting BaLa2Si2S8:Eu2+Thiosilicate Phosphor for LED Lighting", Appl. Mater. Interfaces, vol..7, Dec 2015, pp 40-44, doi: 10.1021/am505613s

[3] S.W.Mhin,J.H.Ryu, K.M.Kim, "Route for Tb3A15O12:Ce3+Colloidal Nanocrystals and Their Luminescent Properties', Nanoscale.Res.Lett, vol.4, Aug2009, pp 888-895,doi:10.1007/s11671-009-9331-9

[4] Aboulaich, M.Michalska, "Ce-Doped YAG Nanophosphor and Red Emitting CuInS2/ZnS Core /Shell Quantum Dots for warm White Light-Emitting Diode with High Color Rendering standard CIE of red light $(0.67,0.33)$, this result indicates that the Sr3B2SiO8: Sm3+ may act as phosphors for the development of W-LEDs under UV/NUV excitation.

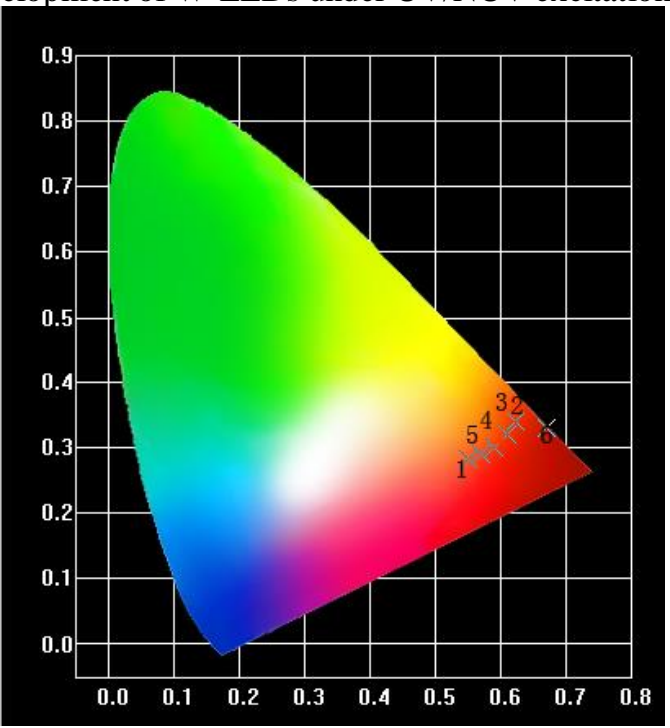

Figure 5. Chromaticity coordinates of $\mathrm{Sr}_{3} \mathrm{~B}_{2} \mathrm{SiO}_{8}: \mathrm{Sm}^{3+}$ (1:(0.55, 0.28), 2: $(0.62,0.34), 3:(0.61,0.32), 4:(0.59,0.30), 5:(0.57,0.29), 6:(0.67$,

$$
0.33) \text { ) }
$$

\section{CONCLUSIONS}

In summary, a series of Sr3B2SiO8: $\mathrm{Sm} 3+$ phosphors have been synthesized by thaditional high temperature solid state reaction. The phosphors Sr3B2SiO8: $\mathrm{Sm} 3+$ excited under the UV region and show orange-red emitting light extended from $550 \mathrm{~nm}$ to $650 \mathrm{~nm}$. In addition, the mechanism of energy transfer between Sm3+ ions is $\mathrm{d}-\mathrm{d}$ interaction, according to the study results based on Dexter's formula and Reisfeld's approximation. The CIE coordinate of $\mathrm{Sr} 3 \mathrm{~B} 2 \mathrm{SiO} 8: 0.03 \mathrm{Sm} 3+$ was calculate to $\mathrm{be}(\mathrm{x}=0.62, \mathrm{y}=0.34)$, which is close to the standard CIE of red light $(0.67,0.33)$. The results showed that Sr3B2SiO8: Sm3+phosphors may be promising phosphor candidate in UV white LEDs.

\section{ACKNOWLEDGMENT}

This present work was financially supported by the Foundation of Chongqing University of Arts and Sciences (R2013CJ09, Y2013CJ30).

Index", Appl. Mater. Interfaces,vol.6, Dec 2014, pp252-258, doi:10.1021/am404108n

[5] K. V. K. Gupta, A. Muley, P. Yadav, C. P Joshi, " Combustion synthesis of YAG:Ce and related phosphors", Applied Physics B" vol.105, Nov2011, pp479-484, doi;10.1007/s00340-011-4685-y

[6] J.Yu, L.Zhou, H.zhang, " Efficient Electroluminescence from New Lanthanide (Eu3+,Sm3+) Complexes”, Inorg.Chem, vol.4, Feb2005, pp1611-1618,doi:10.1021/ic0485561

[7] X.Min,Z.Huang,M.Fang, “ Energy Transfer from Sm3+ to Eu3+ in Red -Emittiing Phosphor LaMgAl11019:Sm3+,Eu3+ for Solar Cells and Near-Ultra Violet White Light -Emitting Diodes", Inorg.Chem, vol.53, Jun2014, pp6060-6065, doi: $10.1021 / \mathrm{ic} 500412 \mathrm{r}$

[8] J. Xu, Z.Ju, “ Na2CaSn2Ge3O12: A Novel Host Lattice for Sm3+-Doped Long-Persistent Phosphorescence Materials 
Emitting Reddish OrangeLight", Inorg.Chem, vol.52, Nov2013, pp13875-13881,doi:10.1021/ic401262w

[9] J.F.Sun,W.L.Zhang, D.Z.Shen, J.Y.Sun, "Intense Red Light Emission of Eu3+-Doped Sr3B2SiO8 for White Light-Emitting Diodes", J. Electrochem. Soc., vol.159, Feb2012, pp107-114, doi: 10.1149/2.073204jes R.D.Shannon.Acta Cryst A32(1976)751

[10] G.Y.Lee,J.Y.Han,W.B.Im, "Novel Blue -Emitting NaxCa1$\mathrm{xSi} 2+\mathrm{XO}$ :Eu2+(x=0.34)Phosphor with High Luminescent
Efficiency for UV-Pumped Light-Emitting Dioles, Inorg.Chem,vol.51, Sep2012, pp10688-10694, doi: 10.1021/ic300956m

[11] D.L. Dexter, "A Theory of Sensitized Luminescence in Solids", J.Chem.Phys., vol.836, May1953, pp836-850, doi:10.1063/1.1699044

[12] D.L. Dexter, J.H. Schulman, "Theory of concentration quenching in inorganic phosphors", J.Chem.Phys., vol.22, 1954, pp1063-1070 doi:10.1063/1.174026. 